\title{
UKnowledge
}

University of Kentucky

UKnowledge

6-13-2013

\section{NFAT5 represses canonical Wnt signaling via inhibition of $\beta$-catenin acetylation and participates in regulating intestinal cell differentiation}

Qingding Wang

University of Kentucky, qingding.wang@uky.edu

Yuning Zhou

University of kentucky, yuning.zhou@uky.edu

Piotr G. Rychahou

University of Kentucky, piotr.rychahou@uky.edu

Chunming Liu

University of Kentucky, chunming.liu@uky.edu

Heidi L. Weiss

University of Kentucky, heidi.weiss@uky.edu

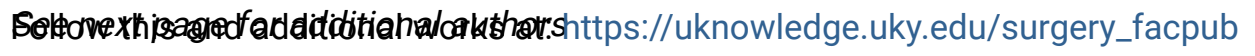

Part of the Surgery Commons

Right click to open a feedback form in a new tab to let us know how this document benefits you.

\section{Repository Citation}

Wang, Qingding; Zhou, Yuning; Rychahou, Piotr G.; Liu, Chunming; Weiss, Heidi L.; and Evers, B. Mark, "NFAT5 represses canonical Wnt signaling via inhibition of $\beta$-catenin acetylation and participates in regulating intestinal cell differentiation" (2013). Surgery Faculty Publications. 9.

https://uknowledge.uky.edu/surgery_facpub/9

This Article is brought to you for free and open access by the Surgery at UKnowledge. It has been accepted for inclusion in Surgery Faculty Publications by an authorized administrator of UKnowledge. For more information, please contact UKnowledge@lsv.uky.edu. 


\section{NFAT5 represses canonical Wnt signaling via inhibition of $\beta$-catenin acetylation}

and participates in regulating intestinal cell differentiation

Digital Object Identifier (DOI)

http://dx.doi.org/10.1038/cddis.2013.202

Notes/Citation Information

Published in Cell Death and Disease, v. 4, no. e671.

Cell Death and Disease is an open-access journal published by Nature Publishing Group. This work is licensed under a Creative Commons Attribution-NonCommercial- ShareAlike 3.0 Unported License. To view a copy of this license, visit http://creativecommons.org/licenses/by-nc-sa/3.0.

Authors

Qingding Wang, Yuning Zhou, Piotr G. Rychahou, Chunming Liu, Heidi L. Weiss, and B. Mark Evers 


\title{
NFAT5 represses canonical Wnt signaling via inhibition of $\beta$-catenin acetylation and participates in regulating intestinal cell differentiation
}

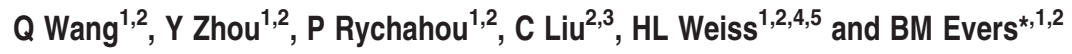

The intestinal mucosa undergoes a continual process of proliferation, differentiation, and apoptosis, which is regulated by multiple signaling pathways. The Wnt/ $\beta$-catenin pathway has a critical role in this process. Previously, we have shown that the calcineurin-dependent nuclear factor of activated T cell (NFAT) is involved in the regulation of intestinal cell differentiation, as noted by the alteration of brush-border enzyme intestinal alkaline phosphatase (IAP) activity. Here, we show that calcineurinindependent NFAT5 interacts with $\beta$-catenin to repress Wnt signaling. We found that overexpression of NFAT5 inhibits, whereas knockdown of NFAT5 increases, TOPflash reporter activity and the expression of Wnt/ $\beta$-catenin target genes, suggesting that NFAT5 inhibits Wnt signaling. In addition, we demonstrated that NFAT5 directly interacts with the C-terminal transactivation domain (TAD) of $\beta$-catenin, inhibits CBP interaction with $\beta$-catenin, and inhibits CBP-mediated $\beta$-catenin acetylation. Moreover, NFAT5 is expressed in the mucosa of human intestine, with the most pronounced staining in the most differentiated region near the epithelial surface. Knockdown of NFAT5 attenuated sodium butyrate (NaBT)-mediated induction of IAP and sucrase activities; overexpression of NFAT5 induced IAP promoter activity. In summary, we provide evidence showing that NFAT5 is a regulator of Wnt signaling. Importantly, our results suggest that NFAT5 regulation of intestinal cell differentiation may be through inhibition of Wnt/ $\beta$-catenin signaling.

Cell Death and Disease (2013) 4, e671; doi:10.1038/cddis.2013.202; published online 13 June 2013

Subject Category: Cancer

The mammalian intestinal mucosa undergoes a process of continual renewal, characterized by active proliferation of stem cells localized near the base of the crypts, progression of these cells up the crypt-villus axis with cessation of proliferation, and subsequent differentiation into one of the four primary cell types (that is, enterocytes, goblet cells, Paneth cells, and enteroendocrine cells). ${ }^{1}$ In the process of differentiation, enterocytes acquire structural features of mature cells, such as microvilli, and express specific gene products, such as intestinal alkaline phosphatase (IAP) and sucrase, brush-border enzymes. ${ }^{2}$ Differentiated enterocytes, which make up the majority of the cells of the gut mucosa, then undergo a process of programmed cell death (that is, apoptosis) and are extruded into the lumen. The cellular mechanisms regulating this tightly regimented process have not been clearly defined. Delineating the molecular factors regulating intestinal proliferation and differentiation is crucial to our understanding of not only normal gut development and maturation, but also aberrant gut growth.

The nuclear factor of activated T cell (NFAT) family proteins are evolutionarily related to the REL (also known as $c-R e l$ )nuclear factor $-\kappa \mathrm{B}$ (REL-NF- $\kappa \mathrm{B})$ family of transcription factors. Five NFAT family members have been identified: NFAT1 (NFATc2 or NFATp), NFAT2 (NFATc1 or NFATc), NFAT3
(NFATc4), NFAT4 (NFATc3 or NFATx), and NFAT5 (tonicity enhancer binding protein (TonEBP)). NFAT proteins contain an amino-terminal transactivation domain (TAD), a regulatory domain (the NFAT homology region (NHR), a highly conserved DNA-binding domain (the Rel-homology domain (RHD), and a C-terminal domain. ${ }^{3,4}$ NFAT proteins are involved in multiple biological processes, ranging from lymphocyte activation and development to cardiac hypertrophy. ${ }^{5,6}$ NFAT has been shown to regulate cell differentiation and development in a number of cell types. ${ }^{7-10}$ NFAT5 has a large C-terminal region that harbors a hypertonicity-sensitive TAD such that hyperosmotic stress can also activate NFAT5. Unlike NFAT1-4 proteins, the NFAT5 subcellular distribution and its phosphorylation state are not altered by calcineurin. NFAT5 has been implicated in diverse processes such as embryonic development, cellular migration, and proliferation. ${ }^{11}$ However, the function of NFAT5 in intestinal cells is largely undefined.

The $\mathrm{Wnt} / \beta$-catenin signaling pathway has a central role for intestinal homeostasis. Wnt/ $\beta$-catenin signaling is essential for maintaining the epithelial proliferative compartment. ${ }^{12}$ Moreover, Wnt signaling controls the differentiation of enterocytes, Paneth cells, goblet cells, and enteroendocrine cells. ${ }^{13-15}$ Two Wnt pathways has been identified: canonical

\footnotetext{
${ }^{1}$ Department of Surgery, Markey Cancer Center, University of Kentucky, Lexington, KY, USA; ${ }^{2}$ Markey Cancer Center, University of Kentucky, Lexington, KY, USA; ${ }^{3}$ Department of Molecular and Cellular Biochemistry, University of Kentucky, Lexington, KY, USA; ${ }^{4}$ Department of Surgery, University of Kentucky, Lexington, KY, USA and ${ }^{5}$ Department of Biostatistics, University of Kentucky, Lexington, KY, USA

${ }^{*}$ Corresponding author: BM Evers, Department of Surgery, Markey Cancer Center, University of Kentucky, 800 Rose Street, CC140, Lexington, KY 40536 0293, USA, Tel: + 502 (859) 323-6556; Fax: + 502 (859) 323 2074; E-mail: mark.evers@uky.edu

Keywords: NFAT5; Wnt; $\beta$-catenin; intestinal cell differentiation

Abbreviations: NFAT, nuclear factor of activated T cell; IAP, intestinal alkaline phosphatase; HEK, human embryonic kidney; GST, glutathione S-transferase; Arm, armadillo; siRNA, small interfering RNA.

Received 18.1.13; revised 07.5.13; accepted 08.5.13; Edited by A Stephanou
} 
or Wnt/ $\beta$-catenin pathway, and a noncanonical pathway or $\beta$-catenin-independent pathway. ${ }^{16}$ In the canonical pathway, Wnt binding leads to the stabilization of $\beta$-catenin, which enters the nucleus to regulate Wnt pathway-target genes. Wnt activation also acts through $\beta$-catenin-independent, noncanonical pathways, such as the planar cell polarity (PCP) pathway and a pathway involving $\mathrm{Ca}^{2+}$ signaling. Mutations in the $\mathrm{Wnt} / \beta$-catenin signaling pathway have been found in most colorectal cancers. ${ }^{16}$

Previously, we have shown that NFATc1, NFATc4, and NFATc3 participate in the regulation of intestinal cell differentiation through regulating PTEN (NFATc1, NFATc4) and REDD1 (NFATc3) expression. In our current study, we found that calcineurin-independent NFAT5 inhibits Wnt $/ \beta$-catenin signaling through interaction with $\beta$-catenin. NFAT5 binds to the $\beta$-catenin transactivation C-terminal domain and prevents CBP interaction with $\beta$-catenin, thus inhibiting $\beta$-catenin acetylation and $\mathrm{Wnt} / \beta$-catenin activation. Furthermore, silencing of NFAT5 attenuates sodium butyrate (NaBT)-induced intestinal cell differentiation, as shown by decreased IAP and sucrase activities. Our results demonstrate that NFAT5 has an important role in the regulation of $\mathrm{Wnt} / \beta$-catenin signaling. NFAT5 may regulate intestinal cell differentiation via the inhibition of the $\mathrm{Wnt} / \beta$-catenin pathway.

\section{Results}

NFAT5 inhibits $\mathbf{W n t} / \boldsymbol{\beta}$-catenin signaling. Previously, we have shown that activation of three calcium-dependent NFAT isoforms, NFATc1, NFATc3, and NFATc4, contributes to intestinal cell differentiation. ${ }^{7,8}$ It has been previously reported that a calcineurin-independent active XNF-AT mutation inhibited anterior development of the primary axis, whereas a dominant negative XNF-AT mutation induced ectopic dorsal axis formation and expression of the canonical Wnt signaling target molecules. ${ }^{17}$ In our current study, we investigated the role of NFAT5, a calcium-independent NFAT isoform, in the regulation of $\mathrm{Wnt} / \beta$-catenin signaling and intestinal cell differentiation. The TOPflash plasmid contains a luciferase reporter under the control of three copies of the TCF/LEF-binding element upstream of the thymidine kinase minimal promoter, and it is specifically regulated by $\mathrm{Wnt} / \beta$ catenin signaling. ${ }^{18}$ We transiently transfected either TOPflash or FOPflash (negative control containing mutated TCF/ LEF-binding sites) plasmids into human embryonic kidney (HEK) 293 cells or the human intestinal cell line Caco-2. As shown in Figure 1a, overexpression of NFAT5 inhibited TOPflash reporter activity. Moreover, knockdown of NFAT5 increased TOPflash reporter activity in HEK293 and Caco-2 cells, and enhanced Wnt3a-stimulated TOPflash reporter activity in HEK293 cells.

To further confirm the NFAT5 regulation of Wnt signaling, the expression of $\mathrm{Wnt} / \beta$-catenin target genes, SP5 and $c-m y c$, was analyzed by quantitative real time RT-PCR. Consistent with the changes in TOPflash reporter activity, knockdown of NFAT5 increased both basal and Wnt3a-stimulated SP5 and c-myc mRNA expression (Figure 1b). Our results suggest a role of NFAT5 in the regulation of Wnt signaling.
To examine whether NFAT5 inhibits Wnt signaling by regulating $\beta$-catenin nuclear accumulation or localization, we used western blots to analyze cytoplasmic and nuclear $\beta$-catenin levels in HEK293 cells with overexpression of NFAT5 (Figure 1c). Overexpression of NFAT5 did not affect either cytoplasmic or nuclear $\beta$-catenin levels, suggesting that NFAT5 may repress Wnt signaling by regulation of $\beta$-catenin transactivation.

NFAT5 interacts with $\beta$-catenin. Wnt $/ \beta$-catenin signaling can be regulated at multiple steps (for example, regulation of $\beta$-catenin protein levels, $\beta$-catenin nuclear localization, $\beta$-catenin/TCF interaction, or interactions between $\beta$-catenin and transcriptional mediator proteins). ${ }^{19}$ Our results have shown that NFAT5 has no effect on $\beta$-catenin protein levels or its localization (Figure 1c). To determine whether NFAT5 interferes with $\beta$-catenin transactivation, we next assessed NFAT5 interaction with $\beta$-catenin. Myc-tagged NFAT5 and FLAG-tagged $\beta$-catenin were co-transfected into HEK293 cells, and an immunoprecipitation assay was performed. As shown in Figures $2 \mathrm{a}$ and $\mathrm{b}$, the interaction between NFAT5 and $\beta$-catenin was detected. To confirm that endogenous NFAT5 binds $\beta$-catenin, NFAT5 was immunoprecipitated from the lysates of HEK293 or Caco-2 cells, and the presence of $\beta$-catenin in the immunoprecipitate was detected by western blot (Figure 2c). Our data suggest that NFAT5 interacts with $\beta$-catenin, thus repressing $\mathrm{Wnt} / \beta$-catenin activation.

$\beta$-Catenin has several functional domains; the $\mathrm{N}$-terminal domain controls $\beta$-catenin stability, the central armadillo domains interact with TCF/LEF and E-cadherin, and the C-terminal domain interacts with the transcriptional mediator complex and activates gene transcription. ${ }^{20}$ To further analyze which domains of $\beta$-catenin bind NFAT5, we used several $\beta$-catenin deletion mutants (Figure 2 d). HEK293 cells were transfected with these mutants, the Myc-tagged $\beta$-catenin mutant proteins were immunoprecipitated and NFAT5 was detected by western blot. As shown in Figure 2e, The N-terminus deletion mutant (Myc-cat- $\Delta-\mathrm{N}$ ), or the armadillo deletion mutant (Myc-cat- $\Delta$-arm), bound NFAT5, whereas no binding of the C-terminus deletion mutant was detected. These results demonstrate that NFAT 5 binds to the C-terminal domain of $\beta$-catenin protein.

To further confirm the direct interaction between NFAT5 and the $\mathrm{C}$-terminal domain of $\beta$-catenin protein, we performed an in vitro binding assay using purified $\beta$-catenin proteins (Figure 2f). ${ }^{21}$ As shown in Figure $2 \mathrm{~d}, \beta$-catenin WT interacts with NFAT5, as expected. However, in vitro binding assays using $\beta$-catenin C-terminal domain-deletion mutants did not demonstrate pull down of NFAT5 protein. We used glutathione S-transferase (GST) as a negative control and showed no binding to NFAT5. Considering that the C-terminal domain interacts with the transcriptional mediator complex and activates gene transcription, ${ }^{20}$ our results suggest that NFAT5 may suppress the function of the $\beta$-catenin C-terminaltransactivating domain.

NFAT5 inhibits binding between $\beta$-catenin and p300/ CBP. Once Wnt is activated, $\beta$-catenin recruits transcriptional co-activators such as p300/CBP and transactivates 
a

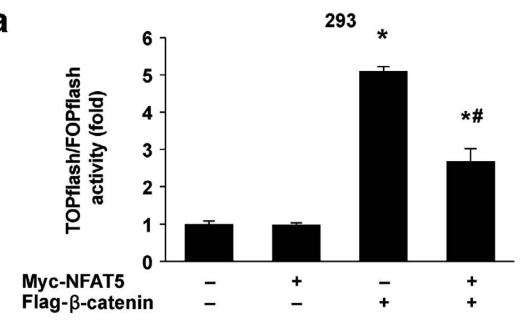

293

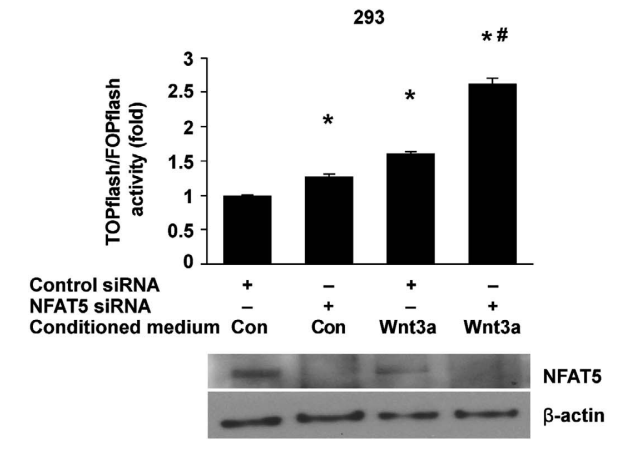

b
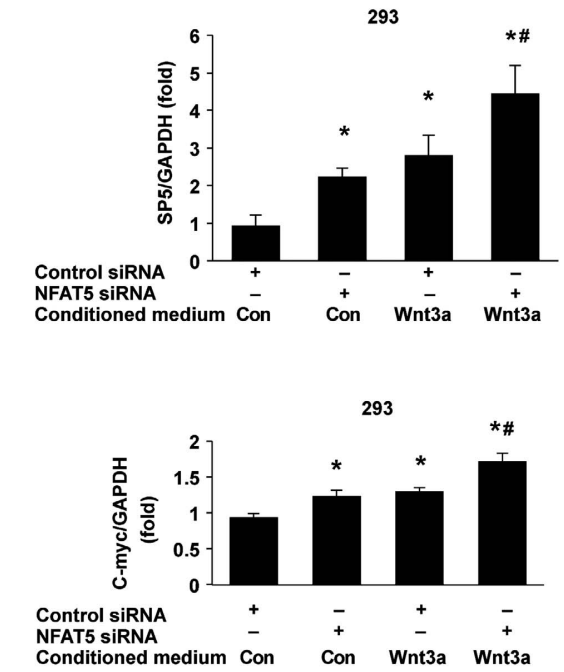
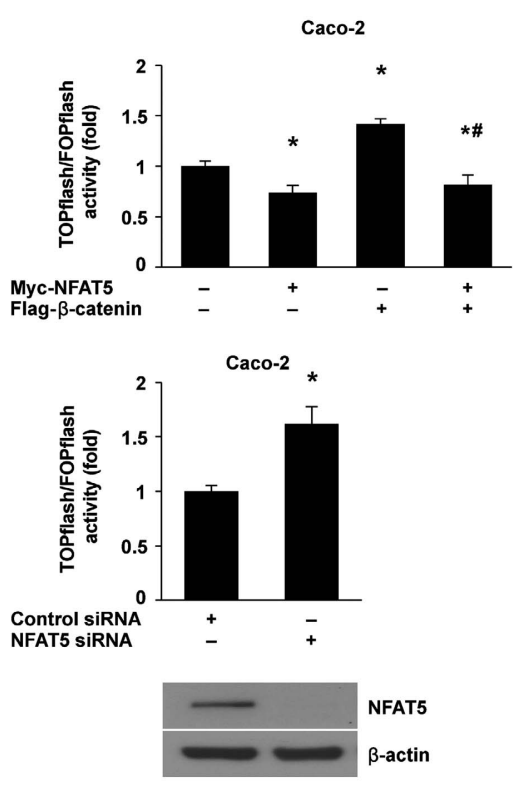

C

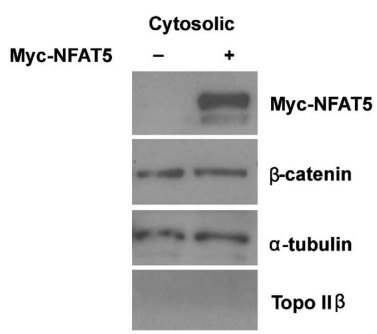

Myc-NFAT5 $\stackrel{\text { Nuclear }}{+}$

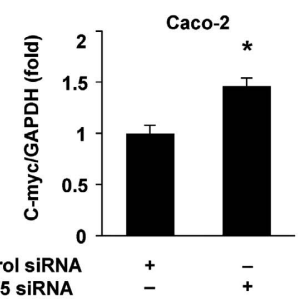

NFAT5 siRNA
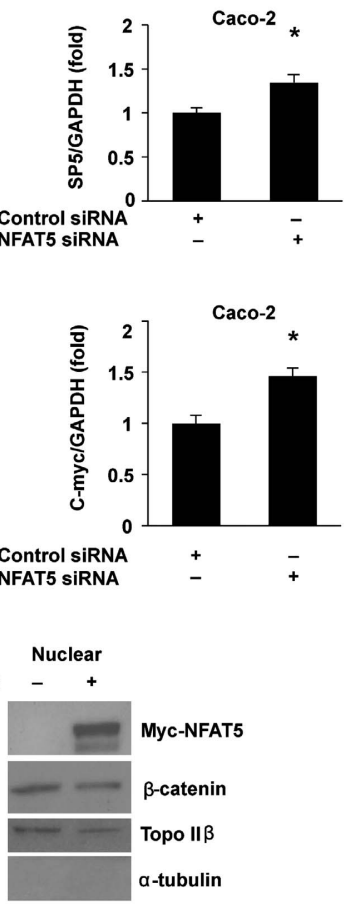

Figure 1 NFAT5 regulates Wnt/ $\beta$-catenin signaling. (a) Effects of NFAT5 on TCF/ $\beta$-catenin reporter activity. HEK293 and Caco-2 cells were co-transfected with TOPflash reporter plasmid or FOPflash-negative control plasmid together with either empty vector, Myc-NFAT5 or Flag- $\beta$-catenin alone or Myc-NFAT5 plus Flag- $\beta$-catenin, and the transfected cells were incubated for $48 \mathrm{~h}$ (upper panels). HEK293 and Caco-2 cells were transfected with control siRNA or siRNA targeting NFAT5. After a 48-h incubation, cells were transfected with TOPflash reporter plasmid or FOPflash-negative control plasmid. Twenty-four hours after retransfection, cells were incubated with control-conditioned medium or Wnt3a-conditioned medium for additional $12 \mathrm{~h}$ (middle panels). Cells were harvested and luciferase activity was measured in the crude cell lysates, as described in Materials and Methods. All results were normalized for transfection efficiency using the pRL-Tk-luc plasmid (Promega). Fold induction corresponds to luciferase activity of positive TOPflash reporter over negative FOPflash reporter. (Data represent mean \pm S.D.; ${ }^{*} P<0.01$ versus control vector or control siRNA; ${ }^{\#} P<0.01 s$ Flag- $\beta$-catenin alone or Wnt3a plus control siRNA, as determined by analysis of variance). The knockdown of NFAT5 was confirmed by western blot (lower panels). (b) Knockdown of NFAT5 increased mRNA expression of SP5 and c-myc, downstream targets of Wnt/ $\beta$-catenin signaling. HEK293 and Caco-2 cells were transfected with control siRNA or siRNA targeting NFAT5. After a 48-h incubation, transfected cells were incubated with control-conditioned medium or Wnt3a-conditioned medium for additional $12 \mathrm{~h}$. Total RNA was extracted, and SP5 and c-myc mRNA levels were determined by real time RT-PCR. (Data represent mean \pm S.D.; ${ }^{*} P<0.01$ versus control siRNA; ${ }^{*} P<0.01$ versus Wnt3a plus control siRNA, as determined by analysis of variance). (c) Overexpression of NFAT5 does not alter cytoplasmic or nuclear $\beta$-catenin levels. HEK293 cells were transfected with empty vector or Myc-NFAT5. After $48 \mathrm{~h}$ of transfection, cytosolic and nuclear proteins were extracted and expression of $\beta$-catenin was detected by western blot 
a

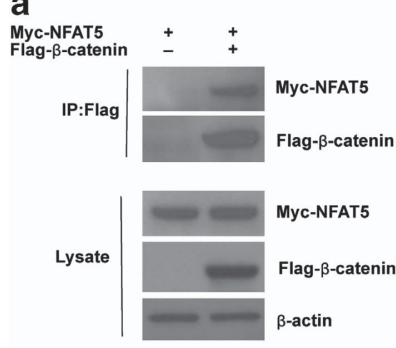

b

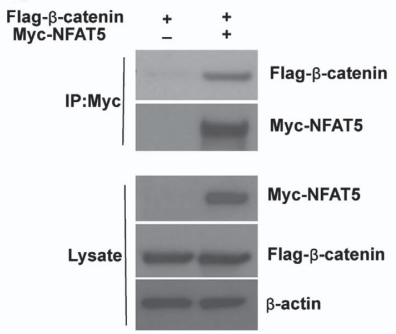

c

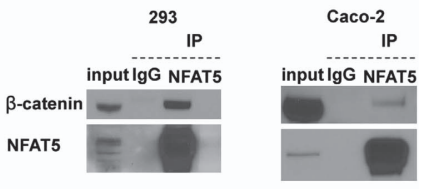

d

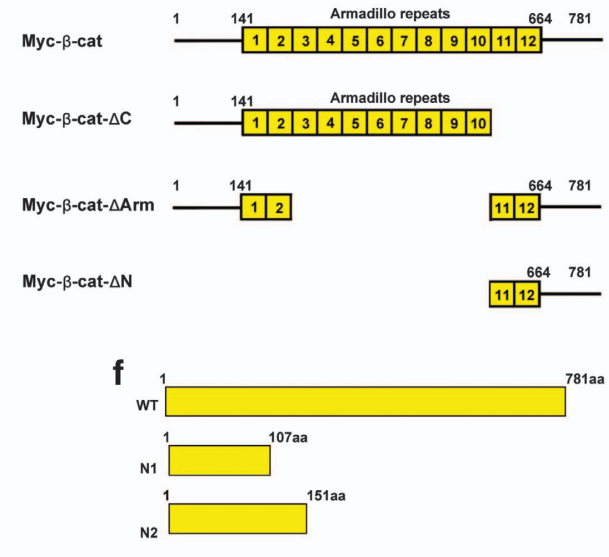

e

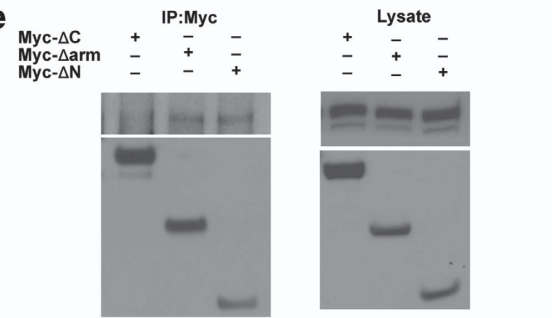

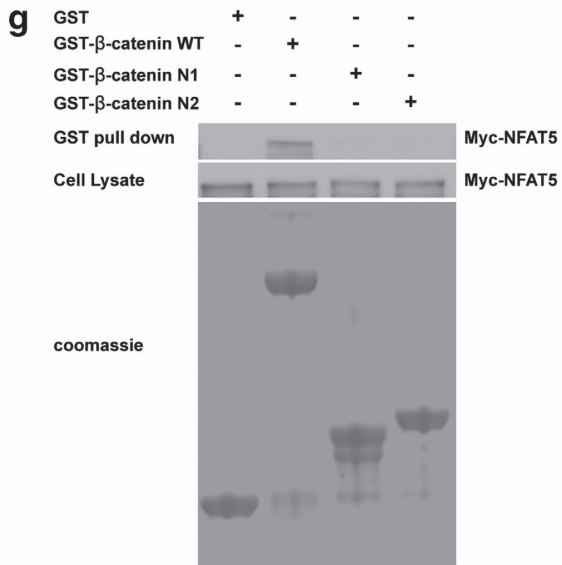

Figure 2 NFAT5 interacts with $\beta$-catenin. (a and $\mathbf{b})$ NFAT5 interacts with $\beta$-catenin. HEK293 cells were co-transfected with Myc-tagged NFAT5 and empty vector or Flagtagged $\beta$-catenin, and incubated for $48 \mathrm{~h}$. After anti-Flag (a) or anti-Myc (b) immunoprecipitation, the presence of NFAT5 and $\beta$-catenin were analyzed using anti-Myc and antiFlag antibodies, respectively. Western blot was performed on cell lysate as a control. (c) Interaction between endogenous NFAT5 and $\beta$-catenin. Endogenous NFAT5 was immunoprecipitated from HEK293 or Caco-2 cells, and Western blot was performed on the eluate. IgG was used as a negative control. (d) Schematic diagram of $\beta$-catenin deletion constructs. The yellow boxes are armadillo repeats. (e) C-terminal-transactivating domains of $\beta$-catenin bind NFAT5. HEK293 cells were co-transfected with Myctagged $\beta$-catenin deletion mutants. Myc-tagged $\beta$-catenin mutants were immunoprecipitated using an anti-Myc antibody. Expression of NFAT5 was analyzed by western blot with an anti-NFAT5 antibody. The expression of these $\beta$-catenin mutants was analyzed by western blot with an anti-Myc antibody. Western blot was performed on cell lysate as a control. (f) Schematic diagram of the wild-type and mutant derivatives of GST- tagged $\beta$-catenin. (g) NFAT5 directly interacts with $\beta$-catenin. HEK293 cells were transfected with Myc-NFAT5 and after $48 \mathrm{~h}$ incubation, lysate was collected and incubated with the GST protein or GST-tagged $\beta$-catenin wide type (WT) or mutant proteins. After several washings, bound proteins were eluted and western blot assay was performed using an anti-Myc antibody

Wnt target gene expression. ${ }^{20}$ To determine whether NFAT5 interferes with the interaction between $\beta$-catenin and $\mathrm{p} 300 /$ CBP, we incubated HA-tagged CBP with GST- $\beta$-catenin protein together with or without Myc-tagged NFAT5 and performed a GST pull-down assay. As shown in Figure 3a, $\beta$-catenin interacts with CBP, as expected. However, overexpression of NFAT5 effectively blocked the ability of $\beta$-catenin to interact with CBP. We used GST as a negative control and showed no binding to CBP (data not shown). Next, we performed a similar experiment using the $\mathrm{CH} 3$ domain of p300 incubated with Flag-tagged $\beta$-catenin, together with or without Myc-tagged NFAT5. As shown in Figure $3 \mathrm{~B}, \beta$-catenin interacts with the $\mathrm{CH} 3$ domain of $\mathrm{p} 300$ and consistent with our previous results, overexpression of
NFAT5 effectively blocked the ability of $\beta$-catenin to interact with the $\mathrm{CH} 3$ domain. Our results from these in vitro experiments indicate that NFAT5 inhibits $\beta$-catenin interaction with $\mathrm{p} 300 / \mathrm{CBP}$.

To test whether NFAT5 could inhibit in vivo binding between $\beta$-catenin and CBP, HEK293 cells were co-transfected with HA-tagged CBP and Flag-tagged $\beta$-catenin or together with Myc-tagged NFAT5 (Figure 3c). After immunoprecipitation using an anti-HA antibody, the interaction between $\beta$-catenin and CBP was detected, as expected. However, overexpression of NFAT5 effectively attenuated this interaction (Figure 3c). Together, these data suggest that NFAT5 interferes with the binding between $\beta$-catenin and p300/ $\mathrm{CBP}$, thus repressing $\mathrm{Wnt} / \beta$-catenin activation. 
a

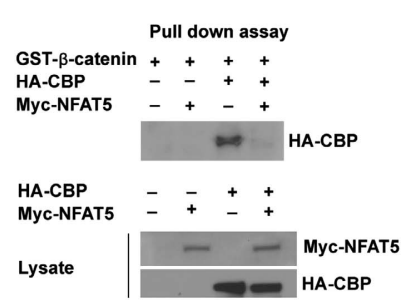

b

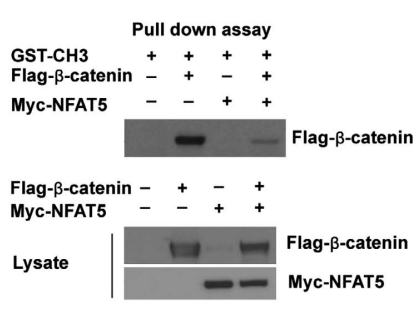

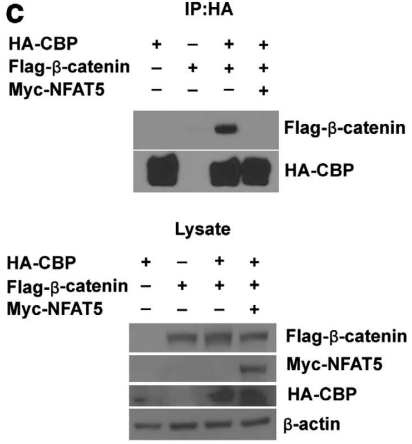

Figure 3 NFAT5 inhibits binding between $\beta$-catenin and p300/CBP. (a) HEK293 cells were transfected with HA-CBP, Myc-NFAT5, or both, and after 48h, lysate was collected and incubated with the GST-tagged $\beta$-catenin protein. After several washings, bound proteins were eluted and an anti-HA western blot assay was performed. (b) HEK293 cells were transfected with Flag- $\beta$-catenin, Myc-NFAT5, or both, and after $48 \mathrm{~h}$, lysate was collected and incubated with the GST-tagged CH3 domain of p300. After several washings, bound proteins were eluted and an anti-Flag western blot assay was performed. (c) HEK293 cells were transfected with HA-tagged CBP and Flag-tagged $\beta$-catenin, with or without Myc-NFAT5 as indicated. Anti-HA immunoprecipitation was performed on the cell lysate, and bound proteins were analyzed via anti-Flag western blot. Whole-cell lysate was analyzed as a control.

NFAT5 inhibits acetylation of $\beta$-catenin by $\mathrm{p} 300 / \mathrm{CBP}$. $\beta$-catenin is acetylated by $\mathrm{p} 300 / \mathrm{CBP} .{ }^{22,23} \mathrm{We}$ found that NFAT5 interferes with interactions between $\beta$-catenin and p300/CBP. To determine whether NFAT5 inhibits $\beta$-catenin acetylation, HEK293 cells were co-transfected with HAtagged $\mathrm{CBP}$ and Flag-tagged $\beta$-catenin, together with or without Myc-tagged NFAT5. Flag-tagged $\beta$-catenin was immunoprecipitated and the acetylated $\beta$-catenin was detected by western blot assay using an anti-acetyl-lysine antibody. As shown in Figure $4, \beta$-catenin was acetylated by CBP as expected, and coexpression of NFAT5 effectively attenuated the acetylation of $\beta$-catenin. Because acetylation of $\mathrm{v}$-catenin results in the increased affinity of $\beta$-catenin to Tcf4 and increased $\beta$-catenin transcriptional activity, ${ }^{22}$ our results demonstrate that NFAT5 interferes with the interaction between $\beta$-catenin and $\mathrm{p} 300 / \mathrm{CBP}$, resulting in decreased $\beta$-catenin acetylation and therefore, inhibits $\beta$-catenin transcriptional activity.

NFAT5 is involved in the regulation of intestinal cell differentiation. Epithelial cells of the intestinal mucosa undergo a continual process of proliferation, differentiation, and apoptosis. The $\mathrm{Wnt} / \beta$-catenin pathway has a critical role in this process. Inhibition of $\mathrm{Wnt} / \beta$-catenin signaling has been shown to induce intestinal cell differentiation. ${ }^{13}$ Human intestinal cell lines Caco-2 and HT29 display a multipotent phenotype, characterized by differentiation into a polarized monolayer and expression of the brush-border enzymes, IAP, and sucrase, when treated with the short-chain fatty acid NaBT ${ }^{24}$ We have shown that NFAT5 inhibited Wnt $/ \beta$ catenin signaling in HEK293 and Caco-2 cells. To determine whether NFAT5 regulates differentiation, we first transfected Caco-2 cells with control siRNA or NFAT5 siRNA and treated with NaBT. Treatment with NaBT increased IAP and sucrase activities (Figure $5 \mathrm{a}$ ); this induction was blocked by transfection with NFAT5 siRNA. To further demonstrate the role of NFAT5 in the regulation of intestinal cell differentiation, Caco-2 cells were transfected with Myc-NFAT5, together with an IAP promoter reporter construct. As shown in Figure 5b, overexpression of NFAT5 resulted in an induction of IAP promoter activity, further suggesting that NFAT5

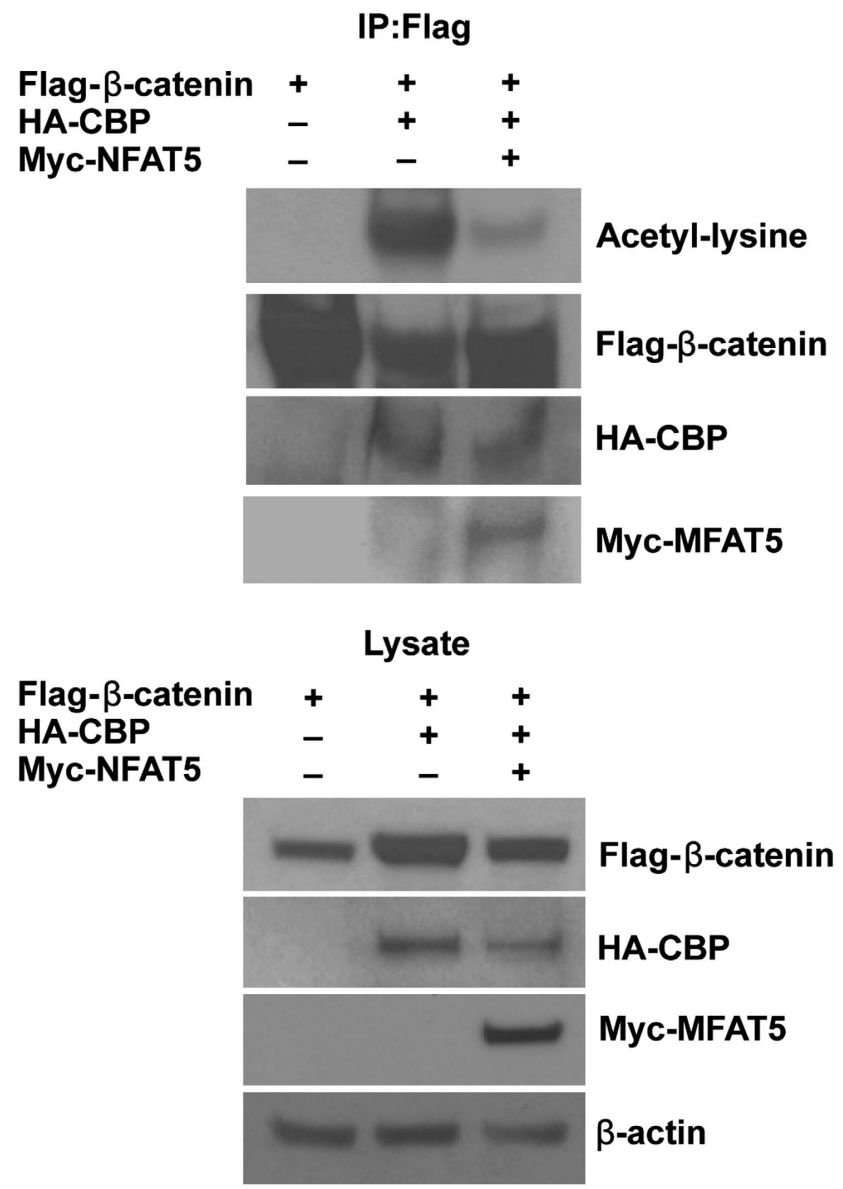

Figure 4 NFAT5 inhibits acetylation of $\beta$-catenin. HEK293 cells were transfected with Flag- $\beta$-catenin, HA-CBP, and Myc-tagged NFAT5 constructs, as indicated. After $48 \mathrm{~h}$, cells were lysed and Flag-tagged $\beta$-catenin was immunoprecipitated using antiFlag antibody. After several washings, the acetylated and total $\beta$-catenin and bound CBP and NFAT5 were analyzed by western blot using antibodies against acetylated lysine, Flag, HA, and Myc (top). Whole-cell lysate was analyzed as control (bottom)

contributes to the differentiation. To determine whether the effect of NFAT5 noted in Caco-2 cells also occurs in other intestinal cell lines, HT29 cells were transfected with control 
a
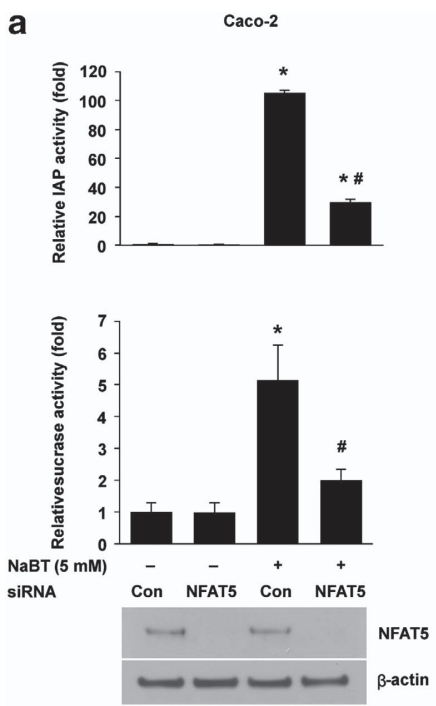

b
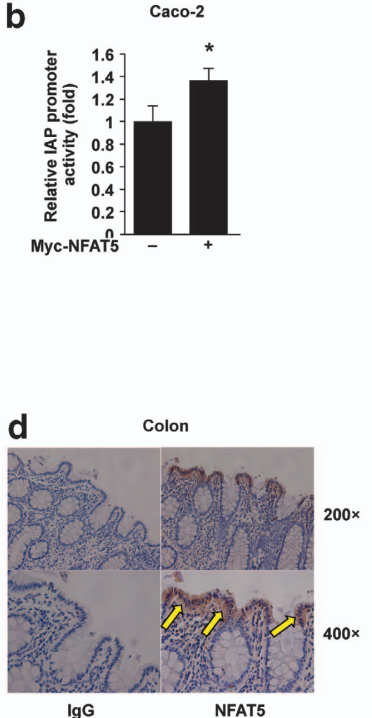

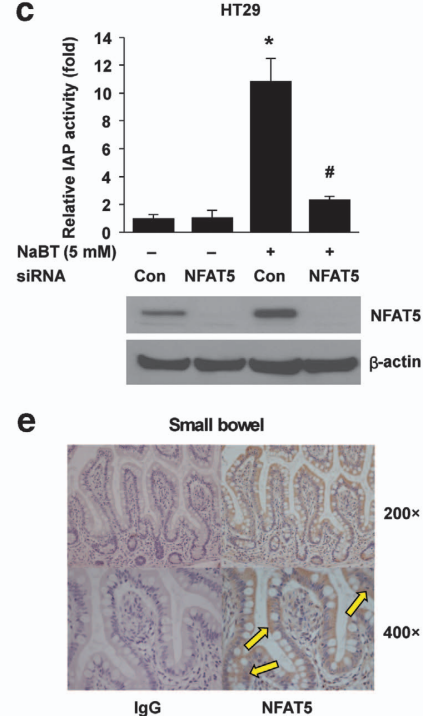

Figure 5 NFAT5 regulation of intestinal cell differentiation. (a) Knockdown of NFAT5 attenuated NaBT-induced IAP and sucrase activities in Caco-2 cells. Caco-2 cells were transfected with control siRNA or siRNA targeting NFAT5. After a 24-h incubation, transfected cells were treated with NaBT ( 5 mM) for additional $72 \mathrm{~h}$. Cells were lysed and alkaline phosphatase and sucrase activities were determined. (Data represent mean \pm S.D.; ${ }^{*} P<0.05$ versus control siRNA; ${ }^{\#} P<0.01$ versus NaBT plus control siRNA, as determined by analysis of variance). (b) Overexpression of NFAT5 increased alkaline phosphatase promoter activity in Caco-2 cells. Caco-2 cells were co-transfected with a plasmid containing the intestinal alkaline phosphatase promoter fragment linked to the luciferase reporter gene and Myc-NFAT5 or empty vector. After a 48-h incubation, the transfected cells were harvested and luciferase activity was measured in the crude cell lysates, as described in Materials and Methods. All results were normalized for transfection efficiency using the pRL-Tk-luc plasmid (Promega). (Data represent mean \pm S.D.; ${ }^{*} P<0.01$ versus control). (c) Knockdown of NFAT5 attenuated NaBT-induced IAP activity in HT29 cells. HT29 cells were transfected with control siRNA or siRNA targeting NFAT5. After a 24-h incubation, transfected cells were treated with NaBT (5 mM) for additional $24 \mathrm{~h}$. Cells were lysed and alkaline phosphatase activity was determined. (Data represent mean \pm S.D.; ${ }^{*} P<0.05$ versus control siRNA; ${ }^{\#} P<0.01$ versus NaBT plus control siRNA, as determined by analysis of variance).(d and e) Immunohistochemical analysis of NFAT5 protein expression in normal human colon (d) and small intestine (e). Tissue sections were fixed and stained with primary anti-human NFAT5 antibody. NFAT5 is mainly expressed in the differentiated region (that is, upper crypts and villus; arrows)

siRNA or NFAT5 siRNA, and treated with NaBT. Treatment with NaBT increased IAP activity (Figure $5 \mathrm{c}$ ); this induction was blocked by knockdown of NFAT5. Together, these results demonstrate the regulation of intestinal cell differentiation by NFAT5.

Our findings demonstrate that NFAT5 has an important role in the regulation of the $\mathrm{Wnt} / \beta$-catenin signaling pathway and intestinal cell differentiation. Finally, to determine the location of NFAT5 expression in vivo, sections of normal human colon (Figure $5 \mathrm{~d}$ ) and small intestine (Figure $5 \mathrm{e}$ ) were obtained from adult patients and analyzed. Interestingly, intense staining for NFAT5 was located in the upper part of the crypt in the colon and the villus fraction of the small intestine. Little staining of NFAT5 was noted in the crypt cells of the small intestine. Therefore, these findings demonstrate that NFAT5 is localized specifically in the more differentiated portions of the intestinal mucosa, thus further suggesting the association of NFAT5 with intestinal differentiation.

\section{Discussion}

Our previous studies demonstrated that activation of calciumdependent NFAT contributes to intestinal cell differentiation. ${ }^{8,25}$ In addition, inhibition of $\mathrm{Wnt} / \beta$-catenin signaling induces intestinal cell differentiation. ${ }^{13}$ In this current study, we provide evidence that calcium-independent NFAT5 binds $\beta$-catenin and interferes with the interaction between $\beta$-catenin and $\mathrm{p} 300 / \mathrm{CBP}$. Moreover, we demonstrate that
NFAT5 blocks p300/CBP-mediated acetylation of $\beta$-catenin. As $\beta$-catenin acetylation has an important role in the regulation of $\beta$-catenin stability, transcriptional activity, and the affinity of $\beta$-catenin for Tcf $4,{ }^{22,23,26}$ our results demonstrate that NFAT5 inhibits Wnt $/ \beta$-catenin through the inhibition of $\beta$-catenin acetylation. Consistent with these results, knockdown of NFAT5 attenuates IAP and sucrase enzyme activities, which are markers of enterocyte differentiation. In addition, NFAT5 is mainly expressed in the more differentiated portions of the intestinal mucosa. Taken together, our results suggest that intestinal cell differentiation is regulated by the NFAT5/Wnt/ $\beta$-catenin signaling pathway.

NFAT5 represses $\beta$-catenin signaling, but does not obviously affect $\beta$-catenin protein levels, suggesting NFAT5 regulation of Wnt signaling downstream of $\beta$-catenin. Indeed, we found that NFAT5 specifically interacted with the $\beta$-catenin $C$-terminus, which contains the transcriptional activation domain. The $\beta$-catenin $\mathrm{C}$-terminus interacts with transcriptional mediators such as p300/CBP. ${ }^{20}$ Similar to NFAT5, KLF4 and inhibitor of $\beta$-catenin and Tcf (ICAT), which also bind the $\beta$-catenin $\mathrm{C}$-terminus, inhibit $\beta$-catenin/p300 binding and $\beta$-catenin transcriptional activity. ${ }^{23,27}$ It is likely that NFAT5 may inhibit $\beta$-catenin function by preventing $\beta$-catenin binding to transcription mediators.

Calcium-dependent NFAT has been shown to repress Wnt signaling through RHD binding to Dvl. ${ }^{28}$ As NFAT5 also has an RHD, NFAT5 may also inhibit Wnt signaling via interaction with Dvl. Although we have had difficulty to detect the effect of 
NFAT5 knockdown on the Wnt3a-induced Dvl2 phosphorylation, we have detected the interaction between NFAT5 and Dvl2 (Supplementary Figure 1). Therefore, we cannot exclude that the effect of NFAT5 on Wnt signaling may not be dependent on the NFAT5-Dvl interaction. It is possible that NFAT5 may bind to not only $\beta$-catenin, but also Dvl isoforms and thus inhibit Wnt signaling. Whether Dvl participates in NFAT5 regulation of Wnt signaling remains to be defined.

NFAT5 has been implicated in the signaling pathways regulating cell differentiation of various cell types. ${ }^{29,30}$ Our results showed that NFAT5 inhibits $\beta$-catenin signaling and participates in the regulation of intestinal cell differentiation. Moreover, we showed that NFAT5 is mainly expressed in the upper crypt portion of the colon and the villus fraction of the small intestine. As the cells reach the midcrypt region, $\beta$ catenin/TCF activity is downregulated, and this results in cell cycle arrest and differentiation. ${ }^{31}$ Similarly, KLF4 is expressed in the villi, and the interaction of KLF4 and $\beta$-catenin inhibits $\beta$ catenin transcriptional activity and regulates intestinal homeostasis. ${ }^{13} \mathrm{~A}$ similar physiological pattern may occur with the interaction between NFAT5 and $\beta$-catenin. The intense staining for NFAT5 located in the more differentiated portions of the intestinal mucosa further suggests that NFAT5 contributes to the maintenance of intestinal homeostasis by preventing activation of $\beta$-catenin signaling in differentiated intestinal cells.

We showed evidence for NFAT5 repression of Wnt $/ \beta$-catenin. However, we found that knockdown of NFAT5 did not alter intestinal cell proliferation (Wang QD et al., unpublished data). It has been reported that NFAT5-deficient lymphocytes and renal cells showed decreased proliferation under hypertonic stress, whereas reduction in NFAT5 activity has no apparent alteration of proliferation in isotonic medium. ${ }^{32}$ In addition, NFAT5 has been implicated in the regulation of the moderate osmoticstress-induced expression of several cyclins. ${ }^{33}$ These findings suggest an osmotic-dependent regulation of cell proliferation by NFAT5. NFAT5 is not only regulated by tonicity, but it can also be activated by various tonicity-independent stimuli in hypertonic and isotonic tissues. ${ }^{34}$ How NFAT5 is regulated in the intestinal cells remains to be defined. Moreover, as we detected NFAT5 expression mainly in the differentiated region of human intestinal mucosa, NFAT5 may likely inhibit Wnt/ $\beta$-catenin upon the intestinal cell differentiation and contribute to the maintenance of intestinal homeostasis. Whether NFAT5 has different role in the proliferating and differentiated intestinal cells are yet to be answered.

Mice lacking NFAT5 have dramatically reduced embryonic viability between days E13.5 and E17.5, and significant perinatal lethality due to kidney defects ${ }^{32,35}$ and impaired cardiac development and function. ${ }^{36}$ However, there is no information demonstrating the resultant intestinal phenotypes in these mice. Interestingly, $\mathrm{Ca}^{2+}$ released from the sarcoplasmic reticulum and the removal of cytoplasmic $\mathrm{Ca}^{2+}$ were increased in cardiomyocytes, with NFAT5

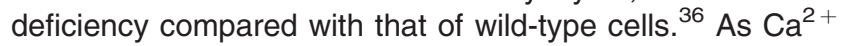
signaling can suppress canonical Wnt activity during axis formation of the Xenopus embryo through the activation of NFAT, ${ }^{17}$ it is possible that NFAT5 represses $\mathrm{Wnt} / \beta$-catenin signaling via the regulation of intracellular $\mathrm{Ca}^{2+}$ signaling. The role of NFAT5 in the differentiation of other cell types has been demonstrated. ${ }^{29,37}$ In this study, we show for the first time that NFAT5 signaling is associated with intestinal cell differentiation. To better understand the role of NFAT5 in intestinal development, it will be necessary to generate intestinal-specific knockout mice to dissect the in vivo function of NFAT5 in the intestine.

The cross-talk between NFAT and Wnt has been shown in some cell types and in Xenopus embryos. ${ }^{17,38,39}$ NFAT can function as the downstream target of Wnt. Apart from the canonical Wnt signaling pathway, activation of which leads to nuclear accumulation of the Lef-Tcf transcriptional coactivator $\beta$-catenin, noncanonical Wnt pathways have been identified, which signal independently of $\beta$-catenin. The Wnt/ $\mathrm{Ca}^{2+} /$ NFAT pathway is a noncanonical Wnt pathway. ${ }^{40}$ In the Wnt/Ca ${ }^{2+} /$ NFAT pathway, Wnt5a-bound Frizzled (FZD), in association with a noncanonical co-receptor, activates phospholipase C (PLC) and increases concentrations of $\mathrm{Ca}^{2+}$, which lead to activation of the $\mathrm{Ca}^{2+}$-calmodulin-dependent protein phosphatase calcineurin and its target, the transcription factor NFAT. ${ }^{39}$ We have shown that calcineurin-independent NFAT5 binds $\beta$-catenin and represses $\mathrm{Wnt} / \beta$-catenin signaling in HEK293 and human intestinal cells. In agreement with our findings, Saneyoshi et al. ${ }^{17}$ showed that calcineurinindependent active XNF-AT mutation inhibits, whereas a lossof-function dominant-negative XNF-AT mutation induces canonical Wnt signaling in Xenopus embryos. In addition, Huang et al. ${ }^{28}$ has shown that calcineurin-dependent NFAT represses canonical Wnt signaling via interaction with Dvl protein. Collectively, our present study as well as those of others identifies NFATs as important repressors for $\mathrm{Wnt} / \beta$ catenin. In contrast to these findings, Adachi et al. ${ }^{41}$ showed that DMSO-induced-TOPflash reporter activity was attenuated in cardiac P19CL6 cells, with overexpression of a dominant negative mutant of NFAT5. These conflicting results may be explained by differences in cell type and the fact that common signaling mechanisms may be interpreted differently, depending on the cellular context.

In conclusion, our results demonstrate that the NFAT5 inhibits $\mathrm{Wnt} / \beta$-catenin signaling through the regulation of $\beta$-catenin acetylation. In the normal intestine, $\beta$-catenin regulates cell proliferation in the base of crypts and inhibits enterocyte differentiation in the upper portion of crypts. NFAT5 is expressed predominantly in differentiated epithelial cells. Our findings suggest that NFAT5 has a critical role in the regulation of intestinal homeostasis through alteration of $\mathrm{Wnt} / \beta$-catenin signaling.

\section{Materials and Methods}

Materials. NaBT, ANTI-FLAG M2 Affinity Gel, Anti-c-Myc Agarose Affinity Gel, Monoclonal Anti-HA - Agarose antibody, and antibodies against Flag, HA, Myc, and $\beta$-actin were purchased from Sigma Chemical Co. (St. Louis, MO, USA). Rabbit Monoclonal anti-c-Myc antibody was from Epitomics Inc. (Burlingame, CA, USA). Rabbit polyclonal anti-NFAT5 antibody was from Thermo Fisher Scientific (Rockford, IL, USA). Rabbit polyclonal anti-tubulin was obtained from Santa Cruz Biotechnology (Santa Cruz, CA, USA). Rabbit anti- $\beta$-catenin, cyclin D1, and antiacetylated-lysine antibodies were purchased from Cell Signaling (Beverly, MA, USA). Mouse anti-human Top $\| \beta$ (clone40/Topoll $\beta$ ) was purchased from BD Biosciences (San Diego, CA, USA). TOPflash (TCF Reporter Plasmid) and negative control reporter FOPflash were from Millipore (Billerica, MA, USA). L cells (ATCC CRL-2648) and L Wnt3A (ATCC CRL-2647) cell line, which were used to obtain control-conditioned medium and Wnt3a-conditioned medium, were from ATCC (Manassas, VA, USA). 
The plasmid encoding human Myc-tagged NFAT5 was from Alex Toker (Boston, MA, USA). Flag- $\beta$-catenin, Myc- $\beta$-catenin mutants, HA-CBP, GST-p300 (CH3), GST- $\beta$-catenin, and GST- $\beta$-catenin mutants have been described previously. ${ }^{21,23,42}$ Flag-Dvl2 was from Addgene (Cambridge, MA, USA). Human NFAT5 and non-targeting control siRNA SMARTpool were purchased from Dharmacon Inc. (Lafayette, CO, USA). siRNA SMARTpool, consisting of four siRNA duplexes, was designed using an algorithm comprised of 33 criteria and parameters that effectively eliminate non-functional siRNA. ${ }^{43}$

Cell culture, transfection, and treatment. HEK293 cells were cultured in Dulbecco's modified Eagle's medium supplemented with $10 \%$ fetal calf serum (FCS) at $37{ }^{\circ} \mathrm{C}$ in $5 \% \mathrm{CO}_{2}$. The human colon cancer cell line Caco-2 was maintained in MEM supplemented with $15 \%$ of FCS. The human colon cancer cell line HT29 was maintained in McCoy's 5A supplemented with 10\% FCS. Cells were transfected with the siRNA duplexes and plasmids by electroporation (Gene Pulser, Bio-Rad, Hercules, CA, USA) and Lipofectamine 2000 (Invitrogen, CA, USA), respectively, as we have described previously. ${ }^{44,45}$ Renilla reporter pRL-null, an internal control to normalize for variation in transfection efficiency, was transfected using Lipofectamine 2000 , and the luciferase activity was determined and normalized to the Renilla activity, as we have described previously. ${ }^{46}$ The TOPflash and FOPflash values were normalized to Renilla, and fold induction was calculated as normalized relative light units of TOPflash divided by normalized relative light units of FOPflash.

Western blot analysis. Nuclear and cytosolic fractions were extracted using NE-PER Nuclear and Cytoplasmic Extraction Reagents kit (Pierce, Rockford, IL, USA). Protein was resolved on a $10 \%$ polyacrylamide gel and transferred to PVDF membranes. Membranes were incubated for $1 \mathrm{~h}$ at room temperature in blotting solution. NFAT5, $\beta$-catenin, acetylated-lysine, Myc-tag, Ha-tag, Flag-tag, and $\beta$-actin were detected with specific antibodies following blotting with a horseradish peroxidase-conjugated secondary antibody, and visualized using an enhanced chemiluminescence $(E C L)$ detection system.

Quantitative real time RT-PCR analysis. Total RNA was extracted and DNase-treated (RQ1, Promega, Madison, WI, USA). Synthesis of CDNA was performed with $1 \mu \mathrm{g}$ of total RNA using the reagents in the TaqMan Reverse Transcription Reagents Kit from ABI (no. N8080234, Foster City, CA, USA). The TaqMan probe and primers for human SP5, c-myc, and GAPDH were purchased from Applied Biosystems (Foster City, CA, USA). Quantitative real time RT-PCR analysis was performed with an Applied Biosystems Prism $7000 \mathrm{HT}$ Sequence Detection System using TaqMan universal PCR master mix, as we have described previously. ${ }^{46}$

GST pull-down. GST-tagged $\mathrm{CH} 3$ and GST-tagged $\beta$-catenin proteins were extracted and purified as we have described previously. ${ }^{21,23}$ Briefly, GST-tagged expression vectors were expressed in Escherichia coli, and the resultant protein was purified with GST beads (glutathione Sepharose; Sigma). GST beads containing purified GST-tagged protein were then incubated with cell lysate at $4{ }^{\circ} \mathrm{C}$ for $1 \mathrm{~h}$; then, beads were washed three times with lysis buffer and boiled in $1 \times$ sodium dodecyl sulfate (SDS) sample buffer, followed by analysis via western blot, as we have described previously. ${ }^{23}$

Enzyme activity assay. Protein was extracted from cells with lysis buffer (20 mm Tris- $\mathrm{HCl}$ (pH 7.5), $150 \mathrm{~mm} \mathrm{NaCl}, 1 \mathrm{~mm} \mathrm{Na} \mathrm{a}_{2}$ EDTA, 1 mm EGTA, 1\% Triton, $2.5 \mathrm{~mm}$ sodium pyrophosphate, $1 \mathrm{~mm} \beta$-glycerophosphate, $1 \mathrm{~mm} \mathrm{Na}_{3} \mathrm{VO}_{4}, 1 \mu \mathrm{g} / \mathrm{ml}$ leupeptin), and the concentrations of the supernatant cell lysates were determined. The supernatant cell lysates $(20 \mu \mathrm{l})$ were used to determine alkaline phosphatase and sucrase activities by commercially available kits from Sigma and BioAssay Systems, respectively, and the data were normalized by the protein amounts used, as previously described. ${ }^{47}$

Immunohistochemical analysis. Formalin-fixed, paraffin-embedded tissue samples of normal human colon and small intestine were used; the samples were taken from the adjacent histologically normal colon and small intestine removed at the time of resection for colon cancer. Tissue was processed for routine immunohistochemical staining using antibodies against human NFAT5. Negative controls (including no primary antibody or isotype-matched mouse immunoglobulin G) were used in each assessment.

Statistical analysis. Descriptive statistics (mean \pm S.D.) were calculated and bar graphs were generated to summarize data across different cell culture experimental conditions. Analysis of reporter activity, IAP and sucrase activities, IAP promoter activity, and qRT-PCR across cell culture groups were performed using analysis of variance model and pairwise comparisons between specific groups identified a priori were performed using contrasts from the model. All statistical analyses were performed using SAS 9.3 (Cary, NC, USA).

\section{Conflict of Interest}

The authors declare no conflict of interest.

Acknowledgements. We thank Heather N Russell-Simmons and Catherine $E$ Anthony for manuscript preparation. This work was supported by grants: P20 CA150343 (UK GI SPORE) and R01 DK48498 from the National Institutes of Health.

1. Cheng $\mathrm{H}$, Leblond $\mathrm{CP}$. Origin, differentiation and renewal of the four main epithelial cell types in the mouse small intestine. V. Unitarian Theory of the origin of the four epithelial cell types. Am J Anat 1974; 141: 537-561.

2. Traber PG. Differentiation of intestinal epithelial cells: lessons from the study of intestinespecific gene expression. J Lab Clin Med 1994; 123: 467-477.

3. Muller MR, Rao A. NFAT, immunity and cancer: a transcription factor comes of age. Nat Rev Immunol 10: 645-656.

4. Hogan PG, Chen L, Nardone J, Rao A. Transcriptional regulation by calcium, calcineurin, and NFAT. Genes Dev 2003; 17: 2205-2232.

5. Molkentin JD, Lu JR, Antos CL, Markham B, Richardson J, Robbins J et al. A calcineurindependent transcriptional pathway for cardiac hypertrophy. Cell 1998; 93: 215-228.

6. Yang TT, Xiong Q, Enslen H, Davis RJ, Chow CW. Phosphorylation of NFATc4 by p38 mitogen-activated protein kinases. Mol Cell Biol 2002; 22: 3892-3904.

7. Zhou $Y$, Wang $Q$, Guo Z, Weiss HL, Evers BM. Nuclear factor of activated T-cell c3 inhibition of mammalian target of rapamycin signaling through induction of regulated in development and DNA damage response 1 in human intestinal cells. Mol Biol Cell 23: 2963-2972.

8. Luo ML, Shen XM, Zhang Y, Wei F, Xu X, Cai Y et al. Amplification and overexpression of CTTN (EMS1) contribute to the metastasis of esophageal squamous cell carcinoma by promoting cell migration and anoikis resistance. Cancer Res 2006; 66: 11690-11699.

9. Santini MP, Talora C, Seki T, Bolgan L, Dotto GP. Cross talk among calcineurin, Sp1/Sp3, and NFAT in control of p21(WAF1/CIP1) expression in keratinocyte differentiation. Proc Natl Acad Sci USA 2001; 98: 9575-9580.

10. Kao SC, Wu H, Xie J, Chang CP, Ranish JA, Graef IA et al. Calcineurin/NFAT signaling is required for neuregulin-regulated Schwann cell differentiation. Science 2009; 323: 651-654.

11. Aramburu J, Drews-Elger K, Estrada-Gelonch A, Minguillon J, Morancho B, Santiago V et al. Regulation of the hypertonic stress response and other cellular functions by the Rel-like transcription factor NFAT5. Biochem Pharmacol 2006; 72: 1597-1604.

12. Pinto D, Gregorieff A, Begthel H, Clevers H. Canonical Wnt signals are essential for homeostasis of the intestinal epithelium. Genes Dev 2003; 17: 1709-1713.

13. Zhang W, Chen X, Kato Y, Evans PM, Yuan S, Yang J et al. Novel cross talk of Kruppel-like factor 4 and beta-catenin regulates normal intestinal homeostasis and tumor repression. Mol Cell Biol 2006; 26: 2055-2064.

14. van Es JH, Jay $P$, Gregorieff $A$, van Gijn ME, Jonkheer S, Hatzis $P$ et al. Wnt signalling induces maturation of Paneth cells in intestinal crypts. Nat Cell Biol 2005; 7: 381-386.

15. Sansom OJ, Reed KR, Hayes AJ, Ireland H, Brinkmann H, Newton IP et al. Loss of Apc in vivo immediately perturbs Wnt signaling, differentiation, and migration. Genes Dev 2004; 18: $1385-1390$.

16. Eisenmann DM. Wnt signaling. In WormBook (ed.), The C. elegans Research Community WormBook, 2005. doi:10.1895/wormbook.1.7.1.

17. Saneyoshi T, Kume S, Amasaki Y, Mikoshiba K. The Wnt/calcium pathway activates NFAT and promotes ventral cell fate in Xenopus embryos. Nature 2002; 417: 295-299.

18. Korinek V, Barker N, Morin PJ, van Wichen D, de Weger R, Kinzler KW et al. Constitutive transcriptional activation by a beta-catenin-Tcf complex in APC - / - colon carcinoma. Science 1997; 275: 1784-1787.

19. Giles $\mathrm{RH}$, van Es JH, Clevers H. Caught up in a Wnt storm: Wnt signaling in cancer. Biochim Biophys Acta 2003; 1653: 1-24.

20. Hecht A, Vleminckx K, Stemmler MP, van Roy F, Kemler R. The p300/CBP acetyltransferases function as transcriptional coactivators of beta-catenin in vertebrates. EMBO J 2000; 19: 1839-1850.

21. Liu C, Kato Y, Zhang Z, Do VM, Yankner BA, He X. beta-Trcp couples beta-catenin phosphorylation-degradation and regulates Xenopus axis formation. Proc Natl Acad Sci USA 1999; 96: 6273-6278.

22. Levy L, Wei $Y$, Labalette $C$, Wu Y, Renard CA, Buendia MA et al. Acetylation of beta-catenin by p300 regulates beta-catenin-Tcf4 interaction. Mol Cell Biol 2004; 24: 3404-3414.

23. Evans PM, Chen X, Zhang W, Liu C. KLF4 interacts with beta-catenin/TCF4 and blocks p300/CBP recruitment by beta-catenin. Mol Cell Biol 30: 372-381. 
24. Wang Q, Wang X, Hernandez A, Kim S, Evers BM. Inhibition of the phosphatidylinositol 3-kinase pathway contributes to HT29 and Caco-2 intestinal cell differentiation. Gastroenterology 2001; 120: 1381-1392.

25. Liu L, Luo Y, Chen L, Shen T, Xu B, Chen W et al. Rapamycin inhibits cytoskeleton reorganization and cell motility by suppressing RhoA expression and activity. J Biol Chem 285: 38362-38373.

26. Winer IS, Bommer GT, Gonik N, Fearon ER. Lysine residues Lys-19 and Lys-49 of beta-catenin regulate its levels and function in T cell factor transcriptional activation and neoplastic transformation. J Biol Chem 2006; 281: 26181-26187.

27. Daniels DL, Weis WI. ICAT inhibits beta-catenin binding to Tcf/Lef-family transcription factors and the general coactivator p300 using independent structural modules. Mol Cell 2002; 10: 573-584.

28. Huang T, Xie Z, Wang J, Li M, Jing N, Li L. Nuclear factor of activated T cells (NFAT) proteins repress canonical Wnt signaling via its interaction with Dishevelled (Dvl) protein and participate in regulating neural progenitor cell proliferation and differentiation. $J$ Bio Chem 286: 37399-37405.

29. O'Connor RS, Mills ST, Jones KA, Ho SN, Pavlath GK. A combinatorial role for NFAT5 in both myoblast migration and differentiation during skeletal muscle myogenesis. J Cell Sci 2007; 120(Pt 1): 149-159.

30. Berga-Bolanos R, Drews-Elger K, Aramburu J, Lopez-Rodriguez C. NFAT5 regulates $T$ lymphocyte homeostasis and CD24-dependent $T$ cell expansion under pathologic hypernatremia. J Immunol 185: 6624-6635.

31. van de Wetering M, Sancho E, Verweij C, de Lau W, Oving I, Hurlstone A et al. The beta-catenin/TCF-4 complex imposes a crypt progenitor phenotype on colorectal cancer cells. Cell 2002; 111: 241-250.

32. Go WY, Liu X, Roti MA, Liu F, Ho SN. NFAT5/TonEBP mutant mice define osmotic stress as a critical feature of the lymphoid microenvironment. Proc Natl Acad Sci USA 2004; 101 10673-10678.

33. Drews-Elger K, Ortells MC, Rao A, Lopez-Rodriguez C, Aramburu J. The transcription factor NFAT5 is required for cyclin expression and cell cycle progression in cells exposed to hypertonic stress. PLoS One 2009; 4: e5245.

34. Halterman JA, Kwon HM, Wamhoff BR. Tonicity-independent regulation of the osmosensitive transcription factor TonEBP (NFAT5). Am J Physiol Cell Physio/302: C1-C8.

35. Lopez-Rodriguez C, Antos CL, Shelton JM, Richardson JA, Lin F, Novobrantseva Tl et al. Loss of NFAT5 results in renal atrophy and lack of tonicity-responsive gene expression. Proc Natl Acad Sci USA 2004; 101: 2392-2397.

36. Mak MC, Lam KM, Chan PK, Lau YB, Tang WH, Yeung PK et al. Embryonic lethality in mice lacking the nuclear factor of activated $T$ cells 5 protein due to impaired cardiac development and function. PLoS One 6: e19186.
37. Wang Y, Ko BC, Yang JY, Lam TT, Jiang Z, Zhang J et al. Transgenic mice expressing dominant-negative osmotic-response element-binding protein (OREBP) in lens exhibit fiber cell elongation defect associated with increased DNA breaks. J Biol Chem 2005; 280: 19986-19991.

38. Yamamoto $F$, Yamamoto $H$. Effect of inhibition of glycogen synthase kinase-3 on cardiac hypertrophy during acute pressure overload. Gen Thorac Cardiovasc Surg 58: 263-264.

39. Gregory MA, Phang TL, Neviani P, Alvarez-Calderon F, Eide CA, O'Hare T et al. Wnt/Ca2 + /NFAT signaling maintains survival of $\mathrm{Ph}+$ leukemia cells upon inhibition of Bcr-Abl. Cancer Cell 18: 74-87.

40. Veeman MT, Axelrod JD, Moon RT. A second canon. Functions and mechanisms of betacatenin-independent Wnt signaling. Dev Cell 2003; 5: 367-377.

41. Adachi A, Takahashi T, Ogata T, Imoto-Tsubakimoto H, Nakanishi N, Ueyama $T$ et al. NFAT5 regulates the canonical Wnt pathway and is required for cardiomyogenic differentiation. Biochem Biophys Res Commun 426: 317-323.

42. Zhang W, Yang J, Liu Y, Chen X, Yu T, Jia J et al. PR55 alpha, a regulatory subunit of PP2A, specifically regulates PP2A-mediated beta-catenin dephosphorylation. J Biol Chem 2009; 284: 22649-22656.

43. Reynolds A, Leake D, Boese Q, Scaringe S, Marshall WS, Khvorova A. Rational siRNA design for RNA interference. Nat Biotechnol 2004; 22: 326-330.

44. Wang $Q$, Wang $X$, Evers BM. Induction of clAP-2 in human colon cancer cells through PKC delta/NF-kappa B. J Biol Chem 2003; 278: 51091-51099.

45. Wang $Q$, Zhou $Y$, Wang $X$, Evers $B M$. Glycogen synthase kinase- 3 is a negative regulator of extracellular signal-regulated kinase. Oncogene 2006; 25: 43-50.

46. Kim S, Domon-Dell C, Kang J, Chung DH, Freund JN, Evers BM. Down-regulation of the tumor suppressor PTEN by the tumor necrosis factor-alpha/nuclear factor-kappaB (NF-kappaB)-inducing kinase/NF-kappaB pathway is linked to a default IkappaB-alpha autoregulatory loop. J Biol Chem 2004; 279: 4285-4291.

47. Wang Q, Zhou Y, Wang X, Evers BM. p27 Kip1 nuclear localization and cyclin-dependent kinase inhibitory activity are regulated by glycogen synthase kinase- 3 in human colon cancer cells. Cell Death Differ 2008; 15: 908-919.

(c) (1) (2) Cell Death and Disease is an open-access journal published by Nature Publishing Group. This work is licensed under a Creative Commons Attribution-NonCommercialShareAlike 3.0 Unported License. To view a copy of this license, visit http://creativecommons.org/licenses/by-nc-sa/3.0/

\section{Supplementary Information accompanies this paper on Cell Death and Disease website (http://www.nature.com/cddis)}

